\title{
"Snowflake" H Mode in a Tokamak Plasma
}

\author{
F. Piras, ${ }^{1,}{ }^{*}$ S. Coda,${ }^{1}$ B. P. Duval, ${ }^{1}$ B. Labit,,${ }^{1}$ J. Marki, ${ }^{1}$ S. Yu. Medvedev, ${ }^{2}$ J.-M. Moret, ${ }^{1}$ \\ A. Pitzschke, ${ }^{1}$ O. Sauter, ${ }^{1}$ and TCV Team \\ ${ }^{1}$ Ecole Polytechnique Fédérale de Lausanne, Centre de Recherches en Physique des Plasmas, \\ Association Euratom-Confédération Suisse, Station 13, CH-1015 Lausanne, Switzerland \\ ${ }^{2}$ Keldysh Institute of Applied Mathematics, Russian Academy of Sciences, Miusskaya 4, 125047 Moscow, Russia
}

(Received 15 June 2010; published 5 October 2010)

An edge-localized mode (ELM) H-mode regime, supported by electron cyclotron heating, has been successfully established in a "snowflake" (second-order null) divertor configuration for the first time in the TCV tokamak. This regime exhibits 2 to 3 times lower ELM frequency and 20\%-30\% increased normalized ELM energy $\left(\Delta W_{\mathrm{ELM}} / W_{p}\right)$ compared to an identically shaped, conventional single-null diverted $\mathrm{H}$ mode. Enhanced stability of mid- to high-toroidal-mode-number ideal modes is consistent with the different snowflake ELM phenomenology. The capability of the snowflake to redistribute the edge power on the additional strike points has been confirmed experimentally.

PACS numbers: 52.55.Fa, 28.52.- s, 52.55.Rk

In the dominant tokamak approach to magneticconfinement nuclear fusion, the divertor configuration has emerged as the preeminent solution to managing power exhaust and core impurity content. In the presence of a magnetic $X$ point, where the poloidal field vanishes, energy and particle losses are channeled primarily into a divertor region that is separated from the confined plasma region. The drawback of this approach is the high power flux impinging on the divertor plates, which may consequently overheat and undergo destructive erosion. This is particularly true during edge localized modes (ELMs) [1-5] occurring in the high-confinement $\mathrm{H}$ mode [6], which cause periodic, violent, and potentially highly damaging ejections of energy and particles onto the divertor surfaces $[7,8]$. Heat flux management in the tokamak divertor and, in particular, the control of ELMs [9-11], remains therefore a primary goal of magnetic fusion research.

A "snowflake" (SF) divertor configuration $[12,13]$ has recently been proposed to reduce the plasma-wall interaction by changing the divertor's poloidal magnetic field topology. The feasibility of this configuration was demonstrated for the first time in the TCV tokamak [14,15]. The $X$ point of a SF configuration features a second-order null point, i.e., both the poloidal magnetic field and its spatial first derivatives vanish. By perturbing this configuration, a $\mathrm{SF}^{+}$configuration or $\mathrm{SF}^{-}$configuration is obtained with two contiguous $X$ points that, for the $\mathrm{SF}^{-}$ configuration, are both located at the separatrix [12]. The distance between the $X$ points normalized to the plasma minor radius $(\sigma)$ parametrizes the proximity to an ideal SF configuration. SF plasmas feature a longer scrape-off layer (SOL) connection length and higher flux expansion in the null-point region compared with conventional singlenull (SN) configuration [14]. Additionally, the increased magnetic shear in the edge region is predicted to influence ELM activity [16,17].
This Letter reports the first $\mathrm{H}$-mode discharges obtained with a $\mathrm{SF}^{+}$divertor. Stationary ELM H modes are obtained and a significant change in the ELM frequency is observed, compared to an equivalent SN divertor plasma, demonstrating the potential of SF plasmas to lead to a new type of highconfinement scenario with better edge properties. In this Letter, we (1) examine the stability and power threshold of the H-mode regime, (2) compare the ELM behavior with an equivalent SN configuration, (3) identify enhanced stability of the kink-ballooning modes consistent with the different $\mathrm{SF}^{+}$ELM dynamics, and (4) examine the $\mathrm{SF}^{+}$configuration power distribution to the additional strike zones.

The $\mathrm{SF}^{+}$configuration studied is shown in Fig. 1(b). The chosen $\sigma \sim 50 \%$ is a compromise between the enhancement of the magnetic properties and the stable sustainment of the configuration by TCV's poloidal coil array and control system. For this study, the $\mathrm{SF}^{+}$is compared with the SN configuration [Fig. 1(a)]. The SN shape is tuned to match the shape and wall separations of the $\mathrm{SF}^{+}$configuration. Differences between the two shapes are significant only in the null-point region where the larger flux expansion of the $\mathrm{SF}^{+}$configuration results in a small modification of the plasma separatrix geometry. For comparison, the last closed flux surfaces (LCFS) of the two moderately shaped configurations (minor radius $a=0.22 \mathrm{~m}$, elongation $\kappa=1.75$, upper triangularity $\delta^{\mathrm{UP}}=0.16$, and lower triangularity $\delta^{\mathrm{LW}}=0.21$ for the $\mathrm{SN}$ and $\delta^{\mathrm{LW}}=0.28$ for the $\mathrm{SF}^{+}$) are shown in Fig. 5(a).

In these scenarios, the $\mathbf{B} \times \boldsymbol{\nabla} B$ ion-drift direction is towards the null point, the plasma current is $300 \mathrm{kA}$, and the toroidal magnetic field at the TCV major radius $\left(R_{0}=0.88 \mathrm{~m}\right)$ is $1.43 \mathrm{~T}$. In addition to the Ohmic power, the plasma is heated with $1.5 \mathrm{MW} \mathrm{X}$-mode electron cyclotron heating $(\mathrm{ECH})$. The ECH beam geometry is shown in Fig. 1: $1 \mathrm{MW}$ is injected at the third electron cyclotron harmonic (X3) from the top of TCV and 0.5 MW is injected 

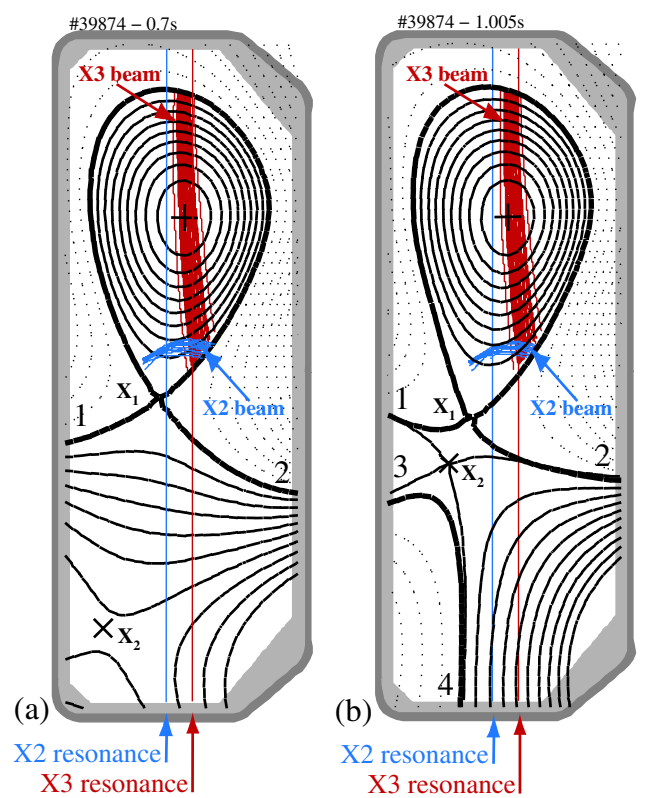

FIG. 1 (color online). SN configuration (a) and $\mathrm{SF}^{+}$configuration (b) with the ECH-X3 beam injected from the top of TCV and the ECH-X2 beam injected from the low-field-side. The positions of the second and third harmonic resonances are represented together with the strike point and null-point positions.

from the low-field side (LFS) at the second harmonic (X2). The absorbed fraction computed by the ray tracing code TORAY-GA [18] is $75 \%$ for the $\mathrm{X} 3$, primarily in the core region, and $100 \%$ for the $\mathrm{X} 2$, localized near the plasma edge.

Figure 2 shows plasma signals during an $\mathrm{H}$ mode with a SN established between 0.28 and $0.7 \mathrm{~s}$, and a $\mathrm{SF}^{+}$stationary phase between 0.8 and $1.64 \mathrm{~s}$. ECH is injected from 0.3 to $1.7 \mathrm{~s}$ [Fig. 2(d)]. This reproducible discharge featuring both $\mathrm{SN}$ and $\mathrm{SF}^{+}$configurations within the same plasma pulse is used to examine the differences and similarities in the H-mode and ELM behaviors. Operated at low plasma density to avoid the ECH cutoff, these plasmas are well below the Ohmic H-mode TCV density threshold and undergo a transition to $\mathrm{H}$ mode only when the $\mathrm{ECH}$ power is applied, identified by the characteristic drop in $\mathrm{H}_{\alpha}$ emission [Fig. 2(a)] and the development of an edge transport barrier in the pressure profile measured by Thomson scattering. The $\mathrm{H}$ mode features steady ELMs, starting immediately after the transition. Figures 2(b) and 2(c) show the temporal evolution of the volume averaged temperature and the line averaged electron plasma density. The volume averaged plasma temperature increases by $\sim 15 \%$ during the $\mathrm{SN}$ to $\mathrm{SF}^{+}$transition as the second $X$ point is moved between the positions shown in Fig. 2. The pedestal temperature does not change [Fig. 5(a)]. The total plasma energy, confinement time, and $\mathrm{H}$ factor increase by $\sim 15 \%$ in the steady-state $\mathrm{SF}^{+}$phase. This relatively small enhancement may be consistent with the variation of the global plasma shape parameters (e.g., the $\sim 30 \%$ increase in the lower triangularity) between $\mathrm{SN}$ and $\mathrm{SF}^{+}$ configurations.

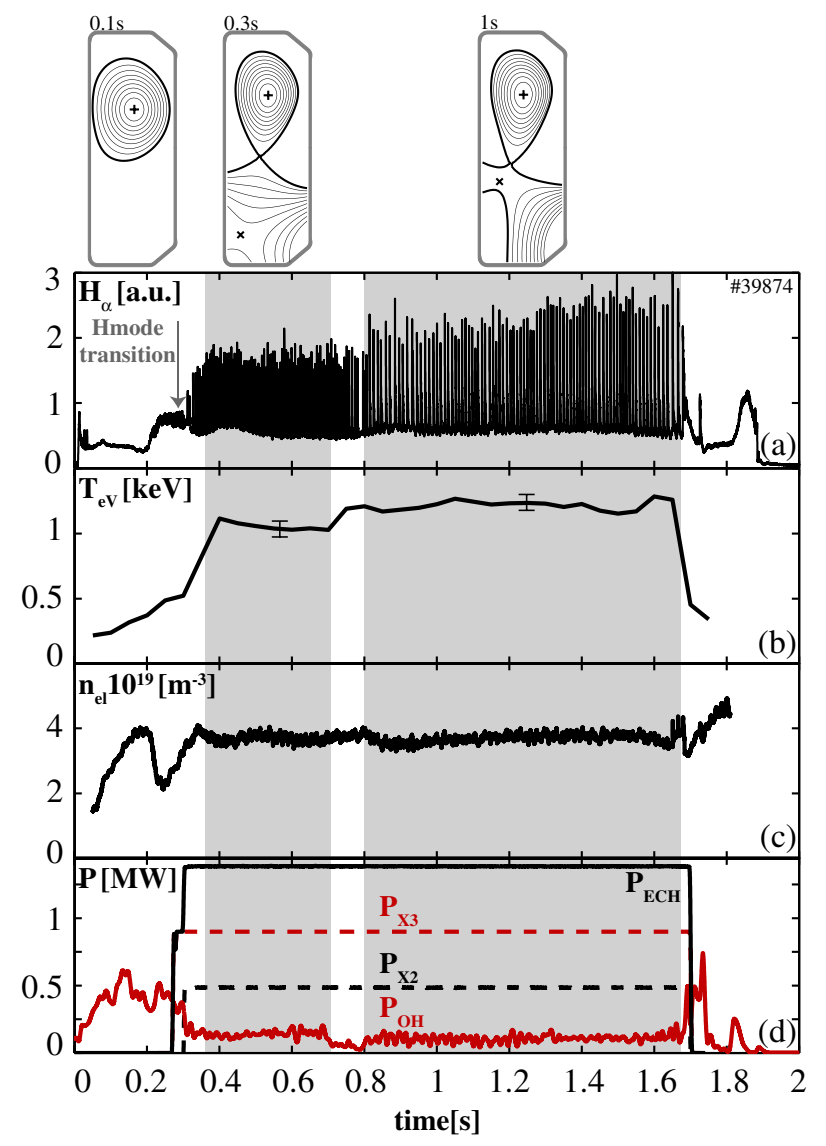

FIG. 2 (color online). (a) $\mathrm{H}_{\alpha}$ edge emission detected by a wide-angle filtered photodiode at the top of the vessel; (b) volume averaged electron plasma temperature; (c) line averaged electron plasma density; (d) Ohmic power (red solid line), $\mathrm{ECH}-\mathrm{X} 2$ power (black dashed line), ECH-X3 power (red dashed line), and total ECH power (black solid line).

The L-H power threshold and the ELM dynamics are crucial properties of $\mathrm{H}$-mode plasmas and their understanding is regarded as an urgent need for controlled fusion. The L-H-mode threshold power for the two configurations is identified with a sequence of discharges with increasing heating power. Figure 3 shows the minimum heating power necessary to establish an $\mathrm{H}$ mode as a function of the volume averaged plasma density $n_{\mathrm{eV}}$. Above $n_{\mathrm{eV}} \sim 5 \times 10^{19} \mathrm{~m}^{-3}$, H-mode access is possible with Ohmic heating power alone. The power threshold is similar for the two configurations, so the change in magnetic topology does not appear to influence the H-mode threshold as one might expect from the increase in shear just inside the separatrix [19]. This is an important result since one of the purposes of snowflake divertors is to open a new regime for ELM $\mathrm{H}$ modes.

In $\mathrm{H}$ modes, ELMs are readily observed as spikes on $\mathrm{H}_{\alpha}$ radiation from the plasma edge. The vertical $\mathrm{H}_{\alpha}$-filtered photodiode, Fig. 2(a), has a field of view that covers all the strike points. A clear change is observed at the $\mathrm{SN}_{-} \mathrm{SF}^{+}$ transition with a reduction in the ELM frequency and an 


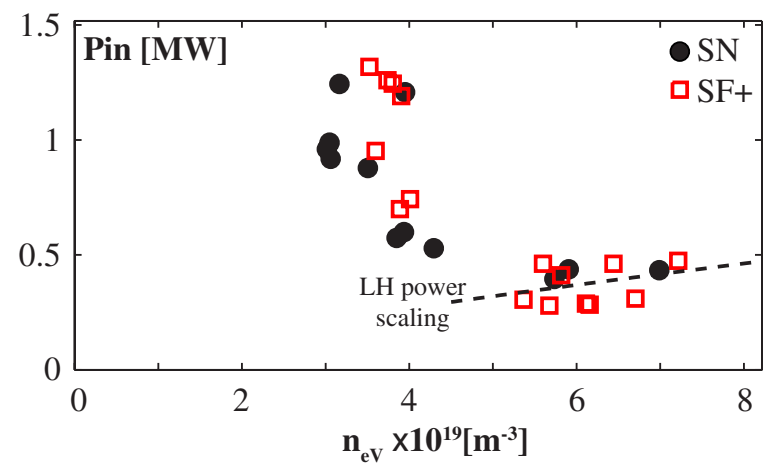

FIG. 3 (color online). H-mode input power threshold vs volume averaged electron plasma density for the SN (black circles) and the $\mathrm{SF}^{+}$(red squares) configurations. The dashed line represents the L-H-mode transition scaling from the international $\mathrm{H}$-mode threshold database [28].

increase in the amplitude of the $\mathrm{H}_{\alpha}$ peaks and their integrated intensity across the ELM by $\sim 30 \%$. The ELMing phases are compared for a sequence of discharges with stepwise increase of the heating power. Figure 4 shows the ELM frequency $\left(\nu_{\mathrm{ELM}}\right)$ versus the input power $\left(P_{\text {in }}\right)$ for both configurations with quantities averaged over a power step held for $200 \mathrm{~ms}$, i.e., long enough compared to the confinement time and to ensure regular ELM activity. The vertical error bars depict the scatter in the computed ELM period and the horizontal error bars are mainly due to $15 \%$ uncertainties in the absorbed fraction of the ECH-X3 power. The ELM frequency increases with heating power $\left(d \nu_{\mathrm{ELM}} / P_{\text {in }}>0\right)$, a behavior typically associated with type I ELM regimes [1]. The high input power compared with $P_{\text {thr }}^{\mathrm{LH}}$ and the absence of a detectable magnetic precursor oscillations also favor a type I classification $[1,20]$. For both configurations, the average energy drop at each ELM $\left(\Delta W_{\mathrm{ELM}}>1.6 \mathrm{~kJ}, \Delta W_{\mathrm{ELM}} / W_{p}>10 \%\right)$ is comparable to, or larger than, that of type I ELMs in other devices [21]. Strikingly, the ELM frequency for the $\mathrm{SF}^{+}$configuration is 2-3 times lower than for the SN configuration, whereas the normalized energy loss at each ELM increases by

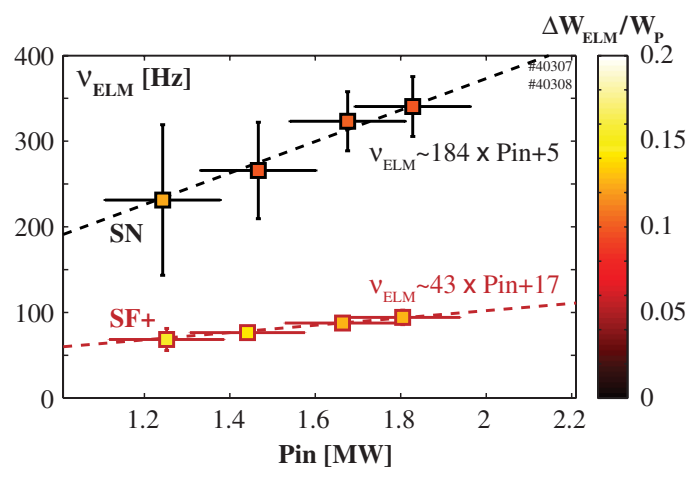

FIG. 4 (color online). ELM frequency variation as a function of the total input power for the $\mathrm{SN}$ and the $\mathrm{SF}^{+}$configurations. The color parametrizes the ELM energy loss normalized to the plasma energy.
20\%-30\%. The ELM behavior is thus indeed strongly modified by the $\mathrm{SF}^{+}$configuration and results in improved plasma performance. The significant reduction of $\nu_{\mathrm{ELM}}$ with a relatively smaller increase of $\Delta W_{\mathrm{ELM}}$ causes a decrease of the normalized ELM power loss $\left(\nu_{\mathrm{ELM}} \times\right.$ $\left.\Delta W_{\text {ELM }} / P_{\text {in }}\right)$, changing from $30 \%$ for the $\mathrm{SN}$ to $\sim 12 \%$ for the $\mathrm{SF}^{+}$configuration.

An analysis of the stability of the plasma edge was undertaken to assess the nature of the ELMs for the two configurations. Figures 5(a) and 5(b) show the temperature and density pedestal profiles for the $\mathrm{SN}$ and the $\mathrm{SF}^{+}$ configurations, demonstrating no significant difference in the pedestal profiles, except for the slightly steeper temperature gradient of the $\mathrm{SF}^{+}$configuration just inside the pedestal region (leading to a $15 \%$ increase in $T_{e 0}$ ). The magnetic shear ( $s=\frac{\rho_{v}}{q} \frac{d q}{d \rho_{v}}$, where $\rho_{v}$ is the square root of the normalized plasma volume) is computed for the two configurations using the CHEASE code [22] and is shown in Fig. 5(b). The edge bootstrap current, calculated from the electron temperature and density profiles [23], is fully
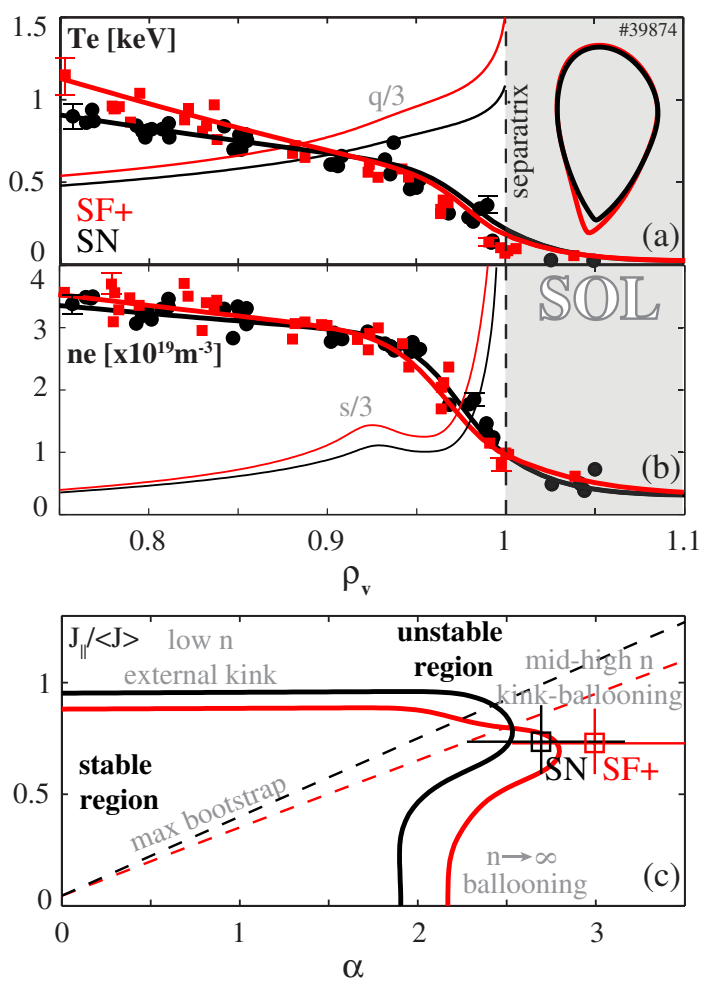

FIG. 5 (color online). (a) Electron temperature and (b) density pedestal profiles for the $\mathrm{SN}$ and the $\mathrm{SF}^{+}$configurations (solid lines) together with the Thomson scattering measurements (dots) as functions of $\rho_{v}$. The error bars of the Thomson scattering measurements for the innermost core and pedestal regions are indicated. Thin lines represent (a) the $q / 3$ profile and (b) the magnetic shear $s / 3$. The LCFS of the two configurations is shown in (a) on the right. (c) Stability diagrams of the SN and the $\mathrm{SF}^{+}$configurations. The collisional bootstrap current is represented by dashed lines together with the experimental points for both configurations (squares). 


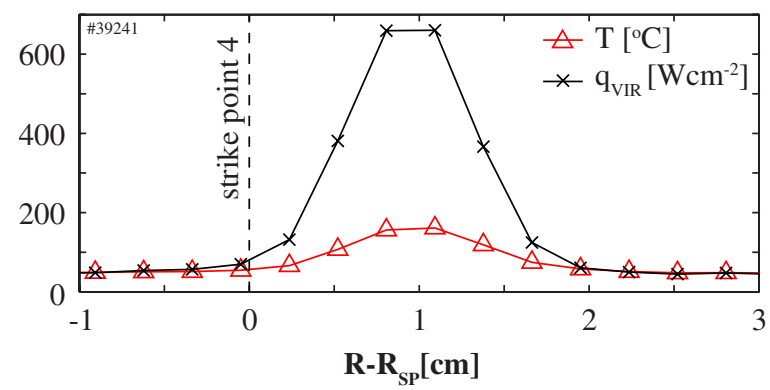

FIG. 6 (color online). Temperature $T$ and heat flux $q_{\text {VIR }}$ profiles at the strike point 4 vs the distance from the strike point at the time of the ELM $\mathrm{H}_{\alpha}$ spike. The profiles are coherently averaged over 30 ELMs.

taken into account. The $\mathrm{SF}^{+}$configuration has higher magnetic shear just inside the plasma separatrix and a slightly lower bootstrap current fraction.

Figure 5(c) shows the MHD stability space diagram of normalized parallel current density in the pedestal $\left(J_{\|} /\langle J\rangle\right)$ vs the normalized pressure gradient $\left(\alpha=\frac{\mu_{0}}{2 \pi^{2}} \frac{d p}{d \psi} \frac{d V}{d \psi} \sqrt{\frac{V}{2 \pi^{2} R_{0}}}\right.$ [24]) computed with the KINX code [25,26]. The squares represent the experimental profiles derived from the Thomson scattering data in Figs. 5(a) and 5(b). The ion temperature is taken to be equal to the electron temperature, as indicated by the charge exchange recombination spectroscopy (CXRS) measurements near the plasma edge. The experimental points for both configurations are close to the kink-ballooning stability boundary, as expected for type I ELM H modes. The second stability region is slightly larger for the $\mathrm{SF}^{+}$configuration, i.e., coupled kinkballooning modes at intermediate-high toroidal mode numbers are more stable. This is a general feature of SF configurations and is confirmed also when calculating the stability diagram with identical profiles.

One of the most unusual features of the SF SOL is the presence of two additional divertor legs [strike points 3 and 4 in Fig. 1(b)]. The properties of the strike point 4 of the $\mathrm{SF}^{+}$configuration are analyzed with a fast vertical infrared (VIR) camera monitoring the floor tiles. Figure 6 shows the coherently averaged ELMs spatial profiles of the heat flux and temperature at the strike point 4 measured with the VIR camera. The peaks of the $\mathrm{H}_{\alpha}$ signal are used as reference for the coherent averaging. A considerable amount of power reaches this strike zone, even though this divertor leg is not directly connected to the main plasma region. Cross-field transport, which is important in the vicinity of the $X$ points, may explain this observation. The total energy deposited at strike point 4 is inferred by integrating the heat flux profile. On average, $\sim 15 \%$ of the total energy lost by the plasma at each ELM (measured with a diamagnetic loop) impinges on strike point 4 , supported by independent calorimetry analysis based on thermocouples in the divertor tiles. No significant broadening of the profiles during the ELM is observed, in contrast with typical observations for the outer strike point of a $\mathrm{SN}$ plasma [27].

In conclusion, a type I ELM H-mode regime has been successfully achieved in a snowflake configuration and compared to an equivalent single-null discharge. The most striking difference is in the ELM frequency, reduced by a factor $2-3$ in the snowflake, while $\Delta W_{\mathrm{ELM}} / W_{\mathrm{P}}$ is increased by only $20 \%-30 \%$. This opens the way to the study of the effect of the new magnetic topology on ELMs.

The authors gratefully acknowledge valuable discussions with S. Alberti, T. Goodman, Y. Martin, R. A. Pitts, and L. Porte. This work was partly supported by the Fonds National Suisse de la Recherche Scientifique.

*francesco.piras@epfl.ch

[1] H. Zohm et al., Plasma Phys. Controlled Fusion 38, 105 (1996).

[2] J. W. Connor et al., Plasma Phys. Controlled Fusion 40, 191 (1998).

[3] H. Takahashi, E. D. Fredrickson, and M. J. Schaffer, Phys. Rev. Lett. 100, 205001 (2008).

[4] L. J. Zheng, H. Takahashi, and E. D. Fredrickson, Phys. Rev. Lett. 100, 115001 (2008).

[5] A. Wingen et al., Phys. Rev. Lett. 104, 175001 (2010).

[6] F. Wagner et al., Phys. Rev. Lett. 49, 1408 (1982).

[7] T. Eich et al., Phys. Rev. Lett. 91, 195003 (2003).

[8] A. Kirk et al., Phys. Rev. Lett. 96, 185001 (2006).

[9] T. E. Evans et al., Phys. Rev. Lett. 92, 235003 (2004).

[10] T. E. Evans et al., Nature Phys. 2, 419 (2006).

[11] Y. Liang et al., Phys. Rev. Lett. 98, 265004 (2007).

[12] D. D. Ryutov et al., Phys. Plasmas 14, 064502 (2007).

[13] D. D. Ryutov et al., Phys. Plasmas 15, 092501 (2008).

[14] F. Piras et al., Plasma Phys. Controlled Fusion 51, 055009 (2009).

[15] F. Hofmann et al., Plasma Phys. Controlled Fusion 36, B277 (1994).

[16] M. V. Umansky et al., Contrib. Plasma Phys. 50, 350 (2010).

[17] P. B. Snyder et al., Phys. Plasmas 9, 2037 (2002).

[18] K. Matsuda et al., IEEE Trans. Plasma Sci. 17, 6 (1989).

[19] K. Toi et al., Phys. Rev. Lett. 64, 1895 (1990).

[20] W. Suttrop et al., Plasma Phys. Controlled Fusion 42, A1 (2000).

[21] A. Loarte et al., Plasma Phys. Controlled Fusion 44, 1815 (2002).

[22] H. Lutjens et al., Comput. Phys. Commun. 97, 219 (1996).

[23] O. Sauter et al., Phys. Plasmas 6, 2834 (1999).

[24] R. J. Groebner et al., Phys. Plasmas 5, 1800 (1998).

[25] L. Degtyarev et al., Comput. Phys. Commun. 103, 10 (1997).

[26] S. Yu. Medvedev et al., Contrib. Plasma Phys. 50, 324 (2010).

[27] J. Marki et al., J. Nucl. Mater. 390-391, 801 (2009).

[28] Y. R. Martin et al., J. Phys. Conf. Ser. 123, 012033 (2008). 